\title{
Bruit généré par la cavitation : modélisation de bruit de bulles sur un profil bidimensionnel, et reconnaissance du type de cavitation par réseaux de neurones artificiels
}

\author{
par G. Lebreuilly, L. Briançon \\ $\mathrm{DCN}$, Bassin d'Essais des Carènes \\ P. Miche \\ L.C.I.A, INSA de Rouen
}

\section{E INTRODUCTION}

Lorsqu'un système matériel subit des variations de volume, ou est en déplacement dans un milieu élastique, il émet un son. Les phénomènes de vaporisation d'un fluide par abaissement local de la pression appelés cavitation n'échappent pas à cette règle. Que ce soient des poches stables ou fluctuantes, des bulles, ou des tourbillons, chaque type de cavitation provoque un bruit particulier.

Mesurés en laboratoire dans des tunnels hydrodynamiques, les signaux acoustiques peuvent être associés visuellement à des figures de cavitation. Cependant sur des installations industrielles, l'information visuelle n'est pas nécessairement accessible. L'idée d'utiliser l'information acoustique, plus facilement mesurable, est une solution tentante, mais s'avère être un problème complexe. Si l'apparition de la cavitation se détecte par l'augmentation du niveau de bruit, il n'existe pas aujourd'hui de système automatique de reconnaissance du type de cavitation.

La première étape suivie par le Bassin d'Essais des Carènes a consisté à produire chaque type de cavitation isolément sur des profils fixes et à étudier le bruit rayonné (Briançon-Marjollet [1], [2]). Les analyses spectrales et statistiques des signaux temporels n'ont pas permis une reconnaissance certaine. Plusieurs facteurs peuvent expliquer la complexité de la classification : chaque type de cavitation fait intervenir du bruit de bulles, les variations de bruit à l'intérieur d'une même classe sont importantes, et la présence de bruit de fond perturbe la reconnaissance. Pourtant, une personne ayant une bonne expérience de ces bruits, est capable de déterminer "à l'oreille" le type de cavitation. En effet, deux personnes habituées au bruit de cavitation ont obtenu lors d'un test $93 \%$ (avec écoute préalable des signaux) et $80 \%$ (aucune écoute) de réponses correctes. Ces constatations ont conduit vers l'utilisation d'outils de classification issus de l'intelligence artificielle : les réseaux de neurones, les études expérimentales de la première étape fournissant une base de données complète de signaux pour chaque type de cavitation.

Au préalable, et afin de mieux connaître les spécificités du bruit généré par une cavitation à bulles, une méthode de simulation a été élaborée. Celle-ci est présentée en première partie de l'article, et est suivie de la reconnaissance du type de cavitation par réseaux de neurones.

\section{II $\square$ SIMULATION DE BRUIT DE CAVITATION A BULLES}

L'objectif de la simulation est de calculer la pression acoustique générée par des bulles de cavitation en un point $\mathrm{M}$ de l'espace. Certaines hypothèses permettent le calcul analytique de la densité spectrale de puissance du signal temporel, en le considérant comme une succession aléatoire d'événements impulsionnels [3], [4], complète cette modélisation en supposant une forme analytique de la pression acoustique rayonnée par la bulle. Cependant, afin de modéliser plus finement le bruit rayonné, il est nécessaire de tenir compte à la fois de la statistique complète des germes ( position, taille initiale, concentration) et de la dynamique de la bulle qui en découle. Nous proposons donc ici une simulation directe utilisant les propriétés suivantes :

- l'équiprobabilité de présence spatiale des bulles dans l'écoulement,

- une répartition en taille de germes obtenue par mesure dans l'installation d'essais.

L'originalité provient d'une part du fait que toutes les lignes de courant pouvant conduire à l'explosion de bulles sont prises en compte et du fait que la population réelle de germes est reproduite.

\subsection{Principe de la méthode}

Le modèle de simulation (Figure 1) de bruit de cavitation à bulles a été appliqué sur un profil bidimensionnel. Un code de calcul est utilisé pour résoudre les équations de Navier-Stokes. Il détermine le champ de pression et de vitesse au voisinage du profil. Pour reconstituer un signal temporel de bruit de cavitation, des germes de tailles aléatoires sont générés à des moments et à une position aléatoires sur une ligne verticale en amont du profil. En estimant les trajectoires des germes (supposées être des lignes de courant), l'évolution en taille des germes présents dans le fluide est calculée en résolvant l'équation de RayleighPlesset. Les variations de volume de la bulle permettent le calcul de la pression rayonnée $\mathrm{P}(\mathrm{M}, \mathrm{t})$ en un point $\mathrm{M}(\mathrm{x}, \mathrm{y})$, les germes étant supposés suffisamment éloignés pour pouvoir négliger leurs interactions. 


\subsection{Modélisations}

L'hypothèse d'indépendance entre les bulles permet de calculer séparément le bruit qu'elles provoquent. $\mathrm{La}$ méthode suivante s'applique à n'importe quel germe injecté en amont du profil.

Le calcul du bruit nécessite au préalable la connaissance du champ de pression et de vitesse autour du profil. Ils sont calculés avec un code de résolution des équations de Navier-stokes pour des conditions non cavitantes. Le profil concerné dans cet article est un NACA009 identique à celui utilisé lors des campagnes de mesures acoustiques sur la veine THEME du Bassin d'Essais des Carènes. Il est localisé entre deux parois situées respectivement à $6 \mathrm{~cm}$ au-dessous et au-dessus. Le maillage de type structuré comporte 102 par 121 quadrilatères. Il intègre le profil et les parois de la veine sur 2 cordes en amont du profil et 5 cordes en aval. Les valeurs du nombre de Reynolds (autour de 1,2E6) imposent de travailler en écoulement turbulent.

La bulle injectée en amont du profil est supposée suivre une ligne de courant sans glissement. Les variations du rayon $\mathrm{R}$ de la bulle, par hypothèse sphérique, le long d'une ligne de courant sont calculées par la résolution numérique de l'équation différentielle de Rayleigh-Plesset avec $R(t=0)=R_{0}$ et dR/dt $\left.\right|_{t=0}=0$.

La pression acoustique temporelle $\mathrm{P}(\mathrm{x}, \mathrm{t})$, générée par la bulle à une distance $\mathrm{x}$, à un temps $\mathrm{t}$, est donnée par la relation [5] :

$$
P(x, t)=\left.\frac{\rho}{4 \pi x} \frac{d^{2} V}{d t^{2}}\right|_{\left(t-\frac{x}{c}\right)}=\frac{\rho}{x}\left(2 R \dot{R}^{2}+\dot{R} R^{2}\right) k_{\left(t-\frac{x}{c}\right)}
$$

\section{$\mathrm{V}=$ Volume de la bulle} $\mathrm{c}=$ Célérité du son dans le fluide

Cette équation représente la solution de l'équation du son en champ lointain ( $x>>$ longueur d'onde) dans le cas d'une fluctuation de volume de gaz, la bulle étant considérée comme une petite source de bruit sphérique (monopole) qui émet à des fréquences dont la longueur d'onde est grande par rapport à la taille de la bulle ( $\mathrm{R}<<$ longueur d'onde). Connaissant la trajectoire de la bulle et ses variations de volume, la pression acoustique peut être calculée en fonction du temps en n'importe quel point de l'espace satisfaisant les hypothèses.

Le calcul du bruit engendré par une bulle peut être relativement long. Il va dépendre de la taille initiale du germe, et de sa position initiale. Pour limiter le temps de simulation d'un signal temporel complet, ces deux variables ont été discrétisées. Le calcul effectué préalablement sur l'ensemble des combinaisons engendre une bibliothèque de pressions acoustiques, qui sera disponible pour une simulation temporelle rapide. Pour limiter la taille de la bibliothèque, le calcul de la pression acoustique générée par un germe n'atteignant pas sa pression critique le
1. Principe de simulation.

long d'une ligne de courant n'est pas effectué. Cette simplification délimite une zone située entre $\mathrm{y}_{\min }$ et $\mathrm{y}_{\max }$ concernée par la cavitation.

\subsection{Générateur de germes}

La configuration des germes est très importante pour la simulation du bruit rayonné. Le générateur de germes est caractérisé par plusieurs variables aléatoires : le rayon $r(k)$ du $k^{\mathrm{cme}}$ germe, l'instant $\mathrm{t}(\mathrm{k})$ où il est injecté, la hauteur $\mathrm{h}(\mathrm{k})$ à laquelle il est injecté. On peut envisager plusieurs types de distributions concernant ces variables, cependant l'hypothèse d'une répartition homogène des germes dans le fluide impose au processus d'injection $t(k)$ d'être poissonien, et une loi uniforme pour $h(k)$. Afin de se rapprocher des configurations d'injection réelles, la distribution des rayons des germes utilisée est identique à celle mesurée par un venturi à ogive centrale dans le tunnel THEME. La concentration $\mathrm{C}$ en germes par $\mathrm{cm}^{3}$ toutes tailles confondues est plus couramment utilisée comme variable représentative. Elle est reliée aux variables suivantes : $L$ largeur du modèle bidimensionnel, $V_{-}$vitesse du fluide, et $\rho_{\mathrm{p}}$ le paramètre du processus de Poisson (nombre moyen de germes par seconde), par la formule:

$$
\rho_{\mathrm{p}}=\mathrm{V}_{\mathrm{o}} * \mathrm{C} * \mathrm{~L} *\left(\mathrm{y}_{\max }-\mathrm{y}_{\min }\right)
$$

\subsection{Résultats de la simulation}

La constitution d'une bibliothèque de pressions acoustiques associée à une vitesse, une pression, une incidence de profil, (environ 4 heures pour cent pressions acoustiques sur un Pentium) permet ensuite des simulations en temps réel de signaux temporels. La Figure 2 présente un exemple de signal temporel simulé (Fréquence d'échantillonage $=10 \mathrm{kHz}$ )

La Figure 3 présente les DSP de signaux simulés se rapportant à trois simulations distinctes. Les cas 1 et 2 présentent le 


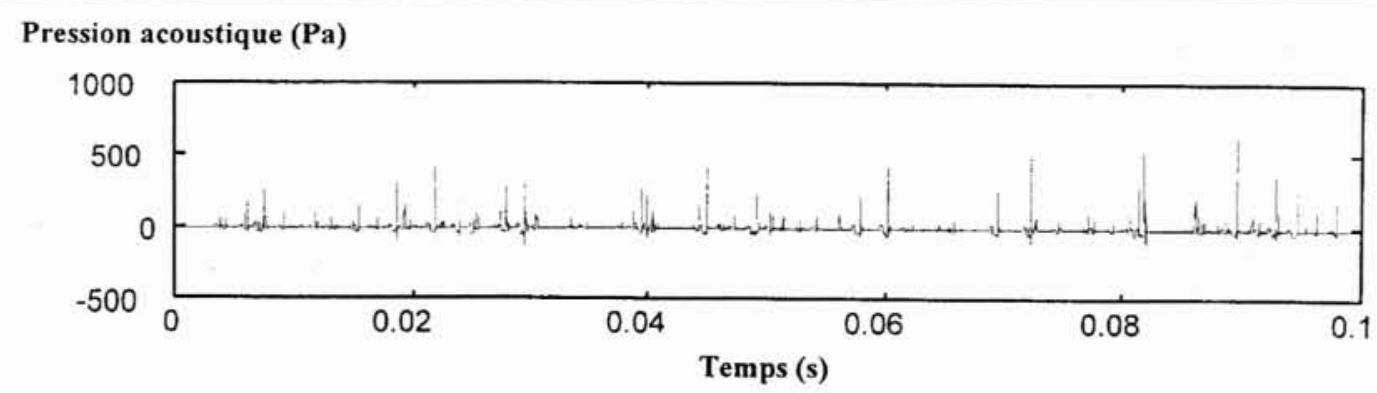

2. Signal temporel de bruit de cavitation avec $\mathrm{V}=12,4 \mathrm{~m} / \mathrm{s}$, Incidence profil $=2^{\circ}, \sigma=0,5$ et $\mathrm{C}=0,7$ germe $/ \mathrm{cm}^{3}$

même nombre de germes par seconde sur une seule ligne de courant (simulation "1D"). Le cas 3 présente une concentration homogène en hauteur (simulation "2D") qui fournit un nombre de germes identique sur la même ligne que les cas 1 et 2 . Tout d'abord le cas bulle unique se rapproche de la théorie de Blake. En effet la DSP présente une bosse correspondant au temps de vie de la bulle (les suivantes sont des harmoniques), et les valeurs des pentes sont comparables à la théorie. L'intégration de plusieurs lignes et plusieurs tailles de bulles modifient sensiblement l'allure de la DSP. La fréquence de la bosse est différente, alors que l'émergence de celle-ci diminue. En intégrant différentes formes de pressions acoustiques dues à des dynamiques différentes, la gamme des temps de vie est élargie, ce qui justifie le développement de cette approche.

Les caractéristiques suivantes ont été relevées à partir de ces spectres :

\section{Variations d'injection}

En augmentant la concentration en germes, le paramètre du processus de Poisson augmente. Les courbes des DSP sur la gamme de fréquence $[0-5 \mathrm{kHz}]$ sont sensiblement parallèles avec une augmentation des niveaux selon la concentration (Figure 4). Elles présentent des bosses plus ou moins marquées, non affectées par les variations d'injection. On observe une

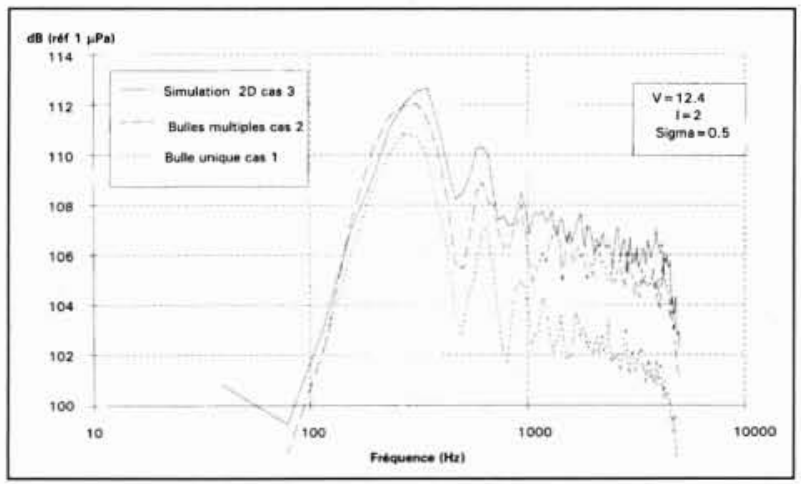

3. DSP des signaux simulés.

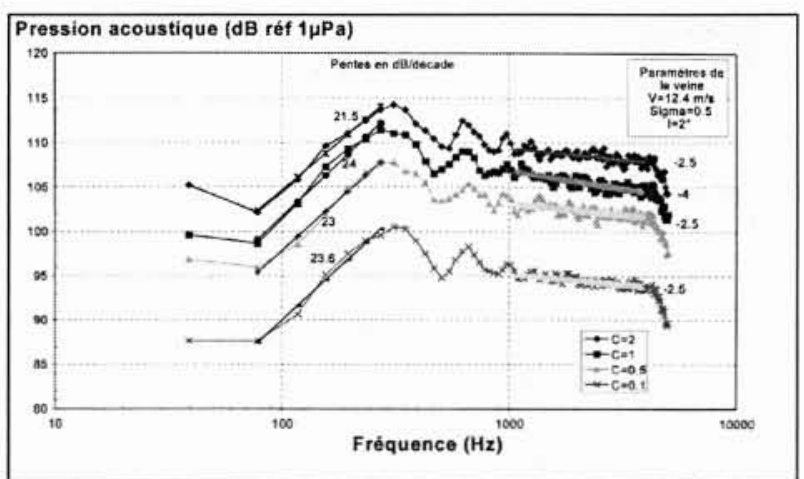

4. DSP des signaux simulés, variations de concentration en germes.

croissance variant peu de $+23 \mathrm{~dB} /$ décade aux fréquences inférieures à la première bosse. Puis une décroissance de $-2,5 \mathrm{~dB} /$ décade aux fréquences supérieures.

La différence de niveau entre 2 concentrations est stable sur toute la bande de fréquence. Elle est proportionnelle au nombre d'événements par seconde. Il y a en effet entre les courbes une différence moyenne de $-3,1,-6,4 \mathrm{et}-14,3 \mathrm{~dB}$ entre $\mathrm{C}=2 \mathrm{~g} / \mathrm{cm}^{3}$ et respectivement $\mathrm{C}=1 \mathrm{~g} / \mathrm{cm}^{3}, \mathrm{C}=0,5 \mathrm{~g} / \mathrm{cm}^{3}$ et $\mathrm{C}=0,1 \mathrm{~g} / \mathrm{cm}^{3}$. Les nombres moyens d'événements par seconde correspondant sont $1225,604,274,50$. Soit des différences de $3,07 \mathrm{~dB}, 6,5 \mathrm{~dB}$, et $13,9 \mathrm{~dB}$.

\section{Variations du nombre de cavitation}

La figure 5 représente des DSP obtenues pour $\mathrm{V}=12,4 \mathrm{~m} / \mathrm{s}$, $\mathrm{C}=1$ germe $/ \mathrm{cm}^{3}$ et une incidence de $2^{\circ}$ pour différents paramètres de cavitation. Les différentes courbes présentent des variations de niveaux et de la fréquence des sommets des bosses. Les tendances relevées (Tableau 2) s'expliquent simplement : lorsque sigma diminue, les temps de vie des germes augmentent, d'où une diminution des fréquences. A même concentration dans l'écoulement, le nombre de germes atteignant leur pression critique augmente, ce qui explique l'aug-

Tableau 1: Caractéristiques des spectres des signaux simulés.

\begin{tabular}{|l|c|c|c|c|}
\hline & $\mathrm{F}_{\max }(\mathrm{Hz})$ & $\begin{array}{c}\text { Croissance } \\
(\mathrm{dB} / \text { décade })\end{array}$ & $\begin{array}{c}\text { Décroissance } \\
(\mathrm{dB} / \text { décade })\end{array}$ & $\begin{array}{c}\text { Emergence } 1^{\text {tre bosse }} \\
\Delta \mathrm{dB}\end{array}$ \\
\hline 1 bulle $\mathrm{R}=120 \mu \mathrm{m}$. cas 1 & 273 & 30,0 & $-2,7$ & 8 \\
\hline Plusieurs bulles/1 ligne. cas 2 & 312 & 31 & $-2,3$ & 6,5 \\
\hline Plusieurs bulles / plusieurs lignes. cas 3 & 351 & 25 & $-3,5$ & 3,5 \\
\hline
\end{tabular}


mentation de niveau. Cependant l'élévation des niveaux est aussi en partie due à l'énergie supérieure des signaux à faible sigma. En effet l'augmentation du nombre de germes cavitants apporte $4 \mathrm{~dB}$ entre $\sigma=0,5$ et $\sigma=0,6$, le reste étant lié à l'augmentation de la pression acoustique générée par chaque germe.

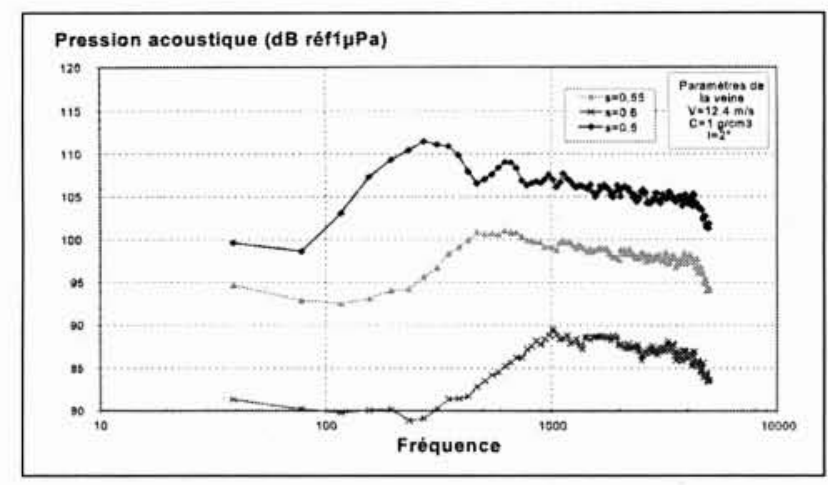

\section{DSP des signaux simulés, variations du nombre} de cavitation.

\subsection{Conclusion}

Les études paramétriques réalisées avec ce modèle prouvent qu'il rend compte des évolutions observées expérimentalement [1]. Les spectres des signaux simulés montrent l'existence de certaines caractéristiques communes à différentes cavitations par bulles. Concernant le signal temporel, on remarque essentiellement son comportement fortement impulsionnel. Ces remarques générales sont utilisées dans la partie suivante pour guider le choix des données d'entrées des réseaux de classification.

\section{III — RÉSEAUX DE NEURONES ET CLASSIFICATION}

La classification est l'une des principales applications des réseaux de neurones. En effet un neurone formel ayant n entrées, muni d'une fonction de transfert de type échelon, est un classificateur binaire. Il sépare l'espace de ces entrées en deux classes par un hyperplan défini par les poids des connexions. L'association de ces classificateurs élémentaires en réseau et l'utilisation de fonctions de transfert non linéaires font des réseaux de neurones un outil bien adapté aux problèmes de classification.

\subsection{Fonctionnement des réseaux de neurones}

L'architecture du réseau choisie pour notre application est de type perceptron multicouche. Elle correspond à un réseau constitué d'un certain nombre de neurones d'entrées (informations données au réseau), d'une couche de sorties donnant la classe, et

Tableau 2: valeurs relevées sur les DSP des signaux simulés.

\begin{tabular}{|c|c|c|c|}
\hline Sigma & 0,5 & 0,55 & 0,6 \\
\hline Fréquence (Hz) & 200 & 500 & 1000 \\
\hline $\begin{array}{c}\text { Nombre d'événement par s } \\
\mathrm{C}=1 \mathrm{~g} / \mathrm{cm}^{3}\end{array}$ & 604 & 387 & 241 \\
\hline Niveau max (dB) & 112 & 101 & 89 \\
\hline
\end{tabular}

d'une ou plusieurs couches cachées permettant de réaliser l'association entrées-sorties désirée [6]. Les connexions sont unidirectionnelles et relient tous les éléments d'une couche à la suivante, aucune autre connexion n'est autorisée. La Figure 6 représente l'architecture utilisée pour la classification des quatre types de cavitation ; les neurones de la couche intermédiaire et de sortie ont des fonctions de transfert de type sigmoïde.

L'algorithme d'apprentissage associé à cette architecture se nomme la rétropropagation du gradient de l'erreur. Il consiste a modifier les poids des connexions dans la direction opposée au gradient de l'erreur quadratique entre la sortie réseau et la sortie désirée.

Au cours de plusieurs essais en tunnel hydrodynamique, des signaux temporels de chaque type de cavitation ont été numérisés avec un appareil de type D.A.T (bande passante $10 \mathrm{~Hz}$ $20 \mathrm{kHz}$, fréquence d'échantillonnage $48 \mathrm{kHz}$ ). Ils correspondent à des conditions de cavitation de type unique observée sur des profils fixes de type NACA. Pour une classification, la base d'apprentissage doit être constituée d'autant d'exemples par type de classe (le plus varié et le plus nombreux possible). Aucun paramètre autre que ceux propres à la cavitation ne doivent permettre la classification : installation, bruit de fond, filtrage,... Concrètement la base d'apprentissage est constituée de 2440 échantillons temporels de 0,1 seconde, représentant 23 Mo de données binaires. Ces échantillons ne sont pas tous appris réellement par le réseau, $26 \%$ de la base de données sont utilisés pour l'algorithme de rétropropagation, $10 \%$ permettent d'éviter le sur-apprentissage par la méthode dite early-stopping [7], et $15 \%$ sont utilisés pour tester les performances du réseau une fois l'apprentissage terminé. Les $49 \%$ d'éléments restant sont des vecteurs d'entrées de type cavitation à bulles et à poches, plus nombreux que les autres classes, et ne peuvent servir qu'à tester le réseau sur ce type de classe séparément.

La sélection du nombre de neurones sur la couche cachée se fait par l'expérience. Le graphe de l'erreur quadratique en fonction de la taille présente une forme parabolique avec un minimum global, auquel correspond le nombre optimal de neurones.

Outre l'erreur quadratique qui est à minimiser par l'algorithme, les performances du réseau sont calculées en interprétant les sorties. La méthode classique consiste à dire que le signal correspond à la classe qui présente l'activation de sortie maximale ("winner takes all"). On utilise également des techniques de seuillage pour décider si le neurone de sortie est actif ou non. Cette dernière interprétation présente l'intérêt de pouvoir activer deux classes simultanément (mélange de deux classes).

\subsection{Entrées utilisées}

Le signal temporel ne peut être utilisé directement comme entrée du réseau pour des problèmes de phase du signal et de taille de réseau. Il est nécessaire d'extraire des paramètres pour caractériser le signal temporel (cf. Erreur! Source du renvoi introuvable.). Ils sont calculés à partir d'un signal temporel réduit afin de s'affranchir des problèmes d'amplitude. Différents types d'entrées ont été testés en s'inspirant notamment de techniques issues de la reconnaissance de la parole [8] et du traitement de l'image :

- Coefficients d'un modèle autorégréssif (AR) : il s'agit d'une modélisation linéaire discrète qui permet de représenter un signal $y(k) k-\varepsilon[1 . . N]$, en un nombre $p$ (ordre du modèle) de coefficients. La méthode consiste à estimer les coefficients $a_{i}$ de manière à minimiser le critère $\mathrm{J}$ défini par les équations suivantes : 


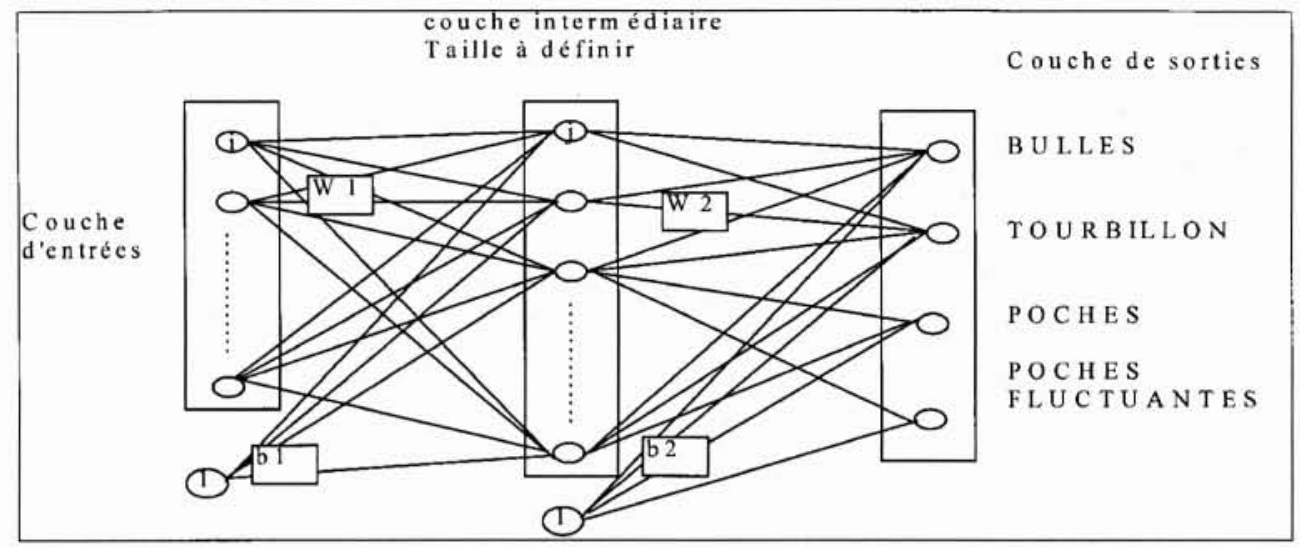

6. Architecture du réseau de neurones.

$$
y(k)=-\sum_{i=1}^{p} a_{i} y(k-i)+e(k) \quad \text { et } J=\sum_{i=1}^{N} e(k)^{2}
$$

L'erreur de prédiction du modèle (Final Prédiction Error selon Akaike) est donnée par :

$$
F P E=\frac{N+p-1}{N-p-1} * J
$$

Cette erreur permet de valider le choix de l'ordre $\mathrm{p}$ du modèle AR. Les p coefficients $a_{i}$ seront les paramètres d'entrée du réseau, ainsi que l'erreur de prédiction finale. L'ordre choisi après plusieurs tests est égal à 9 .

- Les densités spectrales de puissance (DSP) : les caractéristiques du signal utilisées sont les énergies spectrales par bande de $750 \mathrm{~Hz}$ sur [0-24 kHz], soit 32 entrées.

- Les déclivités du signal : une déclivité est un ensemble de points successifs formant un intervalle de signal temporel échantillonné ayant les deux propriétés suivantes : le premier et le dernier point sont des extrema locaux, le signal est strictement monotone sur cet intervalle. Chaque déclivité est décrite par sa longueur $\Delta t$ d'intervalle et son amplitude $\Delta h$. Le signal temporel est représenté par l'histogramme bidimensionnel des couples $(\Delta \mathrm{t}, \Delta \mathrm{h})$. Les hauteurs ont été divisées en 13 classes, les temps en 7. Les 91 cardinaux ne sont pas tous intéressants comme paramètres (certains sont constamment nuls sur la base d'apprentissage). Pour limiter la taille du réseau, les cardinaux des classes ayant une forte variance ont été utilisés, soit 34 d'entre eux. La caractérisation par ce type d'entrées du signal ne pourra être efficace sur les signaux de cavitation qu'après lissage du signal par filtrage passe-bas. Sans cette précaution les entrées seront très sensibles au bruit haute-fréquence.

- Passage par zéro (PPZ) : les paramètres extraits par cette méthode sont les intervalles de temps où le signal est de signe constant. Contrairement à l'exemple précédent, ces paramètres sont plutôt caractéristiques des basses fréquences. Les moments de cette variable aléatoire sont utilisés comme entrées du réseau. Ce type d'entrées n'a pas été testé seul, mais en complément des entrées de type histogramme des déclivités.

Afin d'utiliser l'information présente dans chaque entrée sans augmenter la taille du réseau, et pour éviter certaines redondances d'informations, les entrées ont été codées par différentes méthodes, et présentées en entrées d'un réseau de classification. - Compression par analyse en composante principale (PCA) : Disposant de $\mathrm{N}$ vecteurs de dimension $\mathrm{n}$, le problème consiste à projeter l'ensemble des points sur un sous-espace affine $\mathrm{W}$ de dimension $\mathrm{q}<\mathrm{n}$, de telle sorte que l'on déforme le moins possible le nuage des points. Les données d'entrées étant réduites, la solution du problème consiste à déterminer les axes principaux d'inertie. Le sous espace $\mathrm{W}$ est alors égal à $\left.<\mathrm{X}_{1}, \mathrm{X}_{2}, \ldots, \mathrm{X}_{\mathrm{q}}\right\rangle$ avec $\mathrm{X}_{\mathrm{i}}$ vecteur propre associé à la valeur propre $\lambda_{i}$ de la matrice de covariance des entrées avec $\lambda_{1}>\lambda_{2} \ldots$ $>\lambda_{\mathrm{w}}$. La valeur de q est déterminée de façon arbitraire (seuil de pourcentage d'inertie exprimé par exemple). Les avantages de cette méthode sont l'indépendance des q composantes principales, l'élimination des variables peu ou non significatives, la diminution du nombre de paramètres représentatifs.

- Compression par extraction de paramètre utilisant les méthodes neuronales (NPCA) : l'utilisation d'un réseau est faite pour extraire q paramètres d'un ensemble de $n$ entrées. L'architecture du réseau est de type perceptron multicouche avec 1 couche cachée comportant q neurones. La couche d'entrée et de sortie est constituée de n neurones. L'apprentissage consiste à réaliser la fonction identité. La fonction de transfert de la couche cachée est de type sigmoïdale. Par analogie, on appelle cette technique de traitement une analyse en composantes principales non linéaire [9].

\subsection{Performance des réseaux}

Trois paramètres nous permettent de caractériser les performances des réseaux : l'erreur quadratique atteinte sur les éléments appris, le pourcentage de réponses correctes sur les éléments de validation obtenu par seuillage et par la méthode du tout au gagnant. Les résultats sont comparés avec ceux obtenus par une méthode linéaire, et par une classification par la méthode des $K$ plus proches voisins ( $K P P V$ avec $K=1$ ). Les résultats apportés par un réseau linéaire montrent la nécessité d'utiliser un perceptron multicouche non linéaire. Les deux réseaux utilisant comme entrées l'histogramme des déclivités et les moments d'ordre 1 et 2 des intervalles de temps entre deux passages par zéro se distinguent par leur bonne performance. De plus, ils semblent moins sensibles à la façon d'interpréter les sorties. L'utilisation des DSP montre également de bonnes performances avec un réseau de taille plus réduite.

Une analyse plus fine des performances permet de dégager les tendances suivantes (Tableau 4) : 
Tableau 3. - Performances des réseaux.

\begin{tabular}{|c|c|c|c|c|c|c|}
\hline Type d'entrées & $\begin{array}{c}\% \text { corrects } \\
\text { Réseaux linéaires }\end{array}$ & $\begin{array}{l}\% \text { correct } \\
\text { KPPV. }\end{array}$ & $\begin{array}{c}\text { Taille de la couche cachée/ } \\
\text { nombre de liaisons }\end{array}$ & $\begin{array}{l}\% \text { corrects } \\
\text { Tout au gagnant }\end{array}$ & $\begin{array}{c}\% \text { corrects } \\
\text { seuillage }(0,7)\end{array}$ & Rmserr \\
\hline déclivités & 76,8 & 92,7 & $35 / 1369$ & 93,7 & 92,4 & 0,023 \\
\hline déclivités + PPZ & 76,9 & 92,1 & $100 / 4200$ & 94,5 & 92,7 & 0,0251 \\
\hline $\mathrm{AR}$ & 76,0 & 82 & $320 / 4804$ & 84,9 & 73 & 0,066 \\
\hline DSP & 76,8 & 89,8 & $20 / 760$ & 93,6 & 90,8 & 0,0254 \\
\hline PCA (dimension 18) & 45,05 & 76 & $100 / 2400$ & 76,0 & 64,0 & 0,080 \\
\hline NPCA (comp. 13.1) & 82,8 & 90,3 & $100 / 1600$ & 89,6 & 83,1 & 0,0421 \\
\hline NPCA (comp. 26.2) & 82,0 & 93,7 & $20 / 520$ & 89,9 & 86 & 0,0433 \\
\hline
\end{tabular}

Tableau 4: Performances des réseaux - Analyse par classe. Bu=bulles, To=tourbillon, $P . S=$ poches stables, $P . F=$ poches fluctuantes

\begin{tabular}{|c|c|c|c|c|c|c|c|c|c|c|c|c|c|c|c|c|}
\hline Classe de cavit & \multicolumn{4}{|c|}{ Bulles } & \multicolumn{4}{|c|}{ tourbillon } & \multicolumn{4}{|c|}{ Poches stables } & \multicolumn{4}{|c|}{ Poches fluctuantes } \\
\hline Nombre d'éléments & \multicolumn{4}{|c|}{656} & \multicolumn{4}{|c|}{96} & \multicolumn{4}{|c|}{696} & \multicolumn{4}{|c|}{96} \\
\hline Classes répondues & $\mathrm{Bu}$ & To & P.S & P.F & $\mathrm{Bu}$ & To & P.S & P.F & $\mathrm{Bu}$ & To & P.S & P.F & $\mathrm{Bu}$ & To & P.S & P.F \\
\hline déclivités & 88.4 & 6,2 & 4,5 & 0,9 & 1,0 & 99,0 & 0 & 0 & 1,9 & 0 & 90,8 & 7,3 & 0 & 0 & 2 & 98 \\
\hline déclivités + PPZ & 91,9 & 5,4 & 2,7 & 0 & 1,0 & 99,0 & 0 & 0 & 3,9 & 0,6 & 86,9 & 8,6 & 0 & 0 & 0 & 100 \\
\hline AR & 73 & 2,3 & 6,3 & 18,4 & 1 & 99 & 0 & 0 & 4,3 & 0,4 & 85,7 & 9,6 & 4,1 & 0 & 9,4 & 86,5 \\
\hline DSP & 96,5 & 1,5 & 1,3 & 0,7 & 2,1 & 97,9 & 0 & 0 & 0,7 & 3,4 & 90,8 & 5,1 & 5,2 & 1.0 & 4,2 & 89,6 \\
\hline NPCA (10) & 88,7 & 5,9 & 3,2 & 2,2 & 4,1 & 94,9 & 1 & 0 & 3,9 & 0,7 & 89,7 & 5,7 & 1,0 & 0 & 8,3 & 90,7 \\
\hline NPCA (20) & 93,8 & 2,7 & 2,7 & 0,7 & 2,1 & 97,9 & 0 & 0 & 2,0 & 4,2 & 85,9 & 7,9 & 4,2 & 0 & 7,3 & 88,5 \\
\hline
\end{tabular}

- Les signaux de tourbillons sont les mieux reconnus quel que soit le type d'entrée choisi. Lorsque le réseau se trompe, il indique en majeure partie des signaux de bulles.

- Inversement les signaux bulles sont confondus avec les signaux tourbillon, mais aussi de façon moins fréquente avec des poches stables.

- La classe des poches fluctuantes est le plus souvent confondue avec la classe poches stables et inversement.

\section{IV $\square$ CONCLUSION}

Les techniques neuronales permettent actuellement avec des entrées de type déclivités de reconnaître le type de cavitation sur des profils fixes installés dans la veine THEME avec des performances supérieures à l'oreille humaine. La méthode des KPPV offre également un bon pourcentage de réponses correctes en utilisant des entrées codées par réseau de neurones (NPCA 20). Cependant cette technique nécessite de stocker une grande quantité de données, et l'algorithme de classification est 230 fois plus long. D'autres essais en tunnel de cavitation permettront de tester ces mêmes réseaux pour des signaux de cavitation issus d'une installation différente pour des profils fixes et tournants.

\section{RÉFÉRENCES}

[1] Briançon-Marjol.let.L, Fournier.P "Noise Measurements on Cavitating Hydrofoils: A comparative Study on different Cavitation types"; Second International Symposium on Cavitation, April 1994- Tokyo pp171-177.

[2] Briançon-Marjollet.L, MerLe.L. "Inception, development and noise of a tip vortex cavitation". 21st Symposium on Naval Hydrodynamics. August 1996. Trondheim.

[3] Morozov "Cavitation noise as a train of sound pulses generated at random times". Soviet Physics - Acoustics, Vol. 14 N³, pp. 435-440, Juin 1968.

[4] DE ReFFYE J." Physico-Statistical modelization of the cavitation noise of a propeller". Xth Symposium on hydroacoustics. 1993.

[5] VoKURKA K. "On Rayleigh's model of a freely oscillating bubble. II Results." Czech J Physic 1985.

[6] K. FUNAHASH," On the approximate realization of continuous mappings by neural networks." Neural Networks 2, 1989.

[7] L. PRECHELT, "A set of Neural Network Benchmark Problems and Benchmarking Rules", /pub/papers/techreports/1994-21.ps.Z on ftp.ira.uka.de

[8] Traitement du signal. Volume $7 \mathrm{n}^{\circ} 4$, numéro spécial 1990.

[9] OJA. E "Data compression, Feature Extraction, and Autoassociation in Feedforward Neural Networks". Proc. ICANN-91, Espoo. Finland, Juin 1991, pp 737-745 